\title{
Utilizing DEMATEL for Value-Embedded e-Learning during the COVID-19 Pandemic
}

\author{
Lanndon Ocampo $\mathbb{D}^{1},{ }^{1,2,3}$ Cristy Abarca, ${ }^{1}$ Cristine Abarca, ${ }^{1}$ Ninja Godes, ${ }^{1}$ Esmeralda Pelola, ${ }^{1}$ \\ Margie Pensona, ${ }^{1}$ Magel Niñofranco, ${ }^{1}$ Cherry Ann Itallo, ${ }^{1}$ Cristine Paler, ${ }^{1}$ \\ Celbert Himang $\mathbb{D}^{1},{ }^{1}$ Rebecca Manalastas, ${ }^{1}$ Melanie Himang $\mathbb{D},{ }^{4}$ and Rosein Ancheta Jr. ${ }^{1}$ \\ ${ }^{1}$ Graduate School, Cebu Technological University, Corner M.J. Cuenco Ave., R. Palma St., Cebu City 6000, Philippines \\ ${ }^{2}$ Department of Industrial Engineering, Cebu Technological University, Corner M.J. Cuenco Ave., R. Palma St., \\ Cebu City 6000, Philippines \\ ${ }^{3}$ Center for Applied Mathematics and Operations Research, Cebu Technological University, Corner M.J. Cuenco Ave., \\ R. Palma St., Cebu City 6000, Philippines \\ ${ }^{4}$ College of Information and Communications Technology, Cebu Technological University, Corner M.J. Cuenco Ave., R. Palma St., \\ Cebu City 6000, Philippines
}

Correspondence should be addressed to Lanndon Ocampo; lanndonocampo@gmail.com

Received 29 April 2021; Revised 15 August 2021; Accepted 20 August 2021; Published 31 August 2021

Academic Editor: Ehsan Namaziandost

Copyright ( $) 2021$ Lanndon Ocampo et al. This is an open access article distributed under the Creative Commons Attribution License, which permits unrestricted use, distribution, and reproduction in any medium, provided the original work is properly cited.

\begin{abstract}
Lockdowns of various forms have prompted higher education institutions (HEIs) to suddenly shift from physical face-to-face classes to e-learning environments on an unprecedented scale in recent history. This sudden shift promotes the continuity of the teaching-learning process in HEIs despite the COVID-19 pandemic, at most on the positive side, while bringing forth challenges related to individual learners and academics. This work is based on a recently reported Values-Enhanced Technology Adoption (VETA) model, which incorporates individual values in technology acceptance modeling. Despite offering crucial insights into academics in evaluating e-learning adoption, the current literature suffers from drawbacks. Motivated by addressing these limitations, this work reevaluates the nine constructs of the VETA model using the decision-making trial and evaluation laboratory (DEMATEL). Results indicate that effort expectancy, hedonic motivation, price value, habit, security, tradition, conformity, achievement, power, and hedonism constructs cause performance expectancy, behavioral intention, and social influence. The DEMATEL captures and models the causal relationships between these constructs within an analytical framework, which induces some variations of the recent empirical findings. Finally, the perception of self-achievement among academics drives the intention to adopt e-learning. The findings offered in this work are crucial to the evolving literature of COVID-19 on education, particularly in informing the design of initiatives and measures to enhance e-learning.
\end{abstract}

\section{Introduction}

In curbing COVID-19 transmission, e-learning is deemed to be the immediate alternative to face-to-face classes in universities [1]. At present, after over a year of lockdowns of various forms, academics are coping with e-learning, along with the plethora of lectures, training, webinars, and tutorials on how to effectively use related tools [2]. The transformation from offline to online education brings out some crucial issues in HEIs [3, 4], particularly in developing countries. Higher education institutions (HEIs) in most developing countries are facing various challenges amplified by poor technical infrastructure, pedagogical insufficiencies, and limited resources, along with aspects of culture, practices, skills, competencies, individual values, and attitudes $[5,6]$.

The COVID-19 pandemic highlights abnormal conditions, and an increasing number of published works have 
examined the adoption of distance education through e-learning methodologies during these challenging times [7], including teachers' attributes relevant to information and communication technologies [8], online teaching adoption factors [9], and some exogenous variables (e.g., [10]). The direction of the dynamics of the literature of COVID-19 on HEIs now integrates important constructs that explain the use of e-learning platforms to support distance education [11, 12]. Among other extensions, exploring individual values of learners or users within the context of e-learning amidst the COVID-19 pandemic has not gained much attention, although some insights were reported. The inclusion of social factors as external factors of e-learning acceptance modeling draws interest among scholars in the recent literature (e.g., [13-15]). In this regard, how social factors were integrated into those acceptance models requires some consideration of culture (i.e., an aggregate framework of societal views) or values (i.e., individually held beliefs) [16].

Limited insights on incorporating individual values in e-learning acceptance models were recently offered. Some of these works include Lee et al. [17]; Aparicio et al. [18]; and Tarhini et al. [19]. Mehta et al. [16] bridged these limitations by proposing an extended model incorporating individual values (e.g., conformity, tradition, security, achievement, power, and hedonism). Insights into the values-enhanced model offered by Mehta et al. [16] were explored further by succeeding studies such as evaluating the acceptance of social video platforms (e.g., YouTube) for learning [20], online learning platforms [21, 22], and cloud learning management systems [23]. Brought about by the ongoing COVID-19 pandemic that has since shifted the traditional physical mode of learning to e-learning, the integration of constructs that explain individual values of learners on their acceptance to e-learning or associated platforms has been gaining attention, albeit limited. Inspired by the ValuesEnhanced Technology Adoption (VETA) model of Mehta et al. [16], some reports, including Jameel et al. [24], SitarTăut and Mican [25], Fatima et al. [26], and Sitar-Tăut [27], highlight individual values, at varying degrees, in modeling acceptance of e-learning during the COVID-19 pandemic.

While Mehta et al. [16] reported some empirical findings in the context of two cultural contexts (i.e., The Gambia and the UK), these findings may be idiosyncratic and the effect of COVID-19 pandemic as an emergency may alter acceptance of e-learning. In addition, the viewpoint of academics in e-learning acceptance involving individual values was not explored in recent studies. This agenda is crucial in the teaching-learning process and may pose insights into designing measures that could advance essential aspects of e-learning. Aside from these gaps, a few reservations could be observed from the previous works. First, although popular, the use of structural equation modeling (SEM) (e.g., [28]) as a means for analyzing causal relationships sometimes, if not often, results in some fallacies due to model modification [29]. Second, empirical investigations via SEM involve large samples, and most established models, such as the technology acceptance model (TAM) and the Unified Theory of the Acceptance and Use of Technology (UTAUT), for e-learning require that these samples completely understand the domain context [30]. Finally, with an intention to only model e-learning, identifying crucial constructs of implementing e-learning was not explored. These gaps form the main departure of this work.

Thus, this work aims to advance the methodological process of evaluating the VETA model proposed by Mehta et al. [16] using an expert-opinion-oriented analytical approach, the decision-making trial and evaluation laboratory (DEMATEL). Developed in the 1970 s by the Geneva Research Centre of the Battelle Memorial Institute [31], DEMATEL handles a complex system of elements connected by causal relationships, borrowing its efficacy from the principles of graph theory and linear algebra [32]. It promotes (1) determining the causal relationships among elements in a network (e.g., constructs in a set of constructs) and (2) clustering these elements into the net cause and net effect groups. The DEMATEL is a practical and useful tool for visualizing the structure of complex causal relationships between elements into an intelligible structural model [29]. In the education sector, applications of the DEMATEL are limited, which include strategic management [33], performance evaluation $[34,35]$, and e-learning $[36,37]$. Note that this list is not intended to be comprehensive. Various attempts were reported on the use of the DEMATEL approach in evaluating some popular models, including TAM [38, 39], UTAUT [30, 40], and Decomposed Theory of Planned Behavior [41, 42]. Sheng-Li et al. [43] provided a thorough review of the methodologies and applications of the DEMATEL during the recent decade.

With the efficacy of the DEMATEL in modeling causal relationships (e.g., [44, 45]), this work advances the proposed VETA model of Mehta et al. [16] by offering a different methodological perspective. In this work, the 13 constructs of the VETA model are adopted in constructing a structural model using DEMATEL. Contrary to SEM, the proposed methodology provides the following innovations: (1) hidden causal relationships among constructs are uncovered, (2) aside from the direct effects of one construct to another, it takes further consideration into the indirect effects among constructs, which would then generate total relations, (3) it identifies critical constructs for decisionmaking, (4) it provides a better understanding on complicated and intertwined problems, instrumental in an exploratory mode of analysis, and (5) it addresses technical problems (e.g., e-learning) with limited sample sizes. This work advances the evolving literature of COVID-19 on education by effectively providing a structural model based on expert analysis on the use of e-learning platforms of academics. The remainder of this paper is arranged as follows: Section 2 presents a brief background of the DEMATEL. The proposed methodology is described in Section 3. Section 4 details the results and the findings. The paper ends with a conclusion and discussion of future work in Section 5.

\section{Review of the Related Literature}

The onset of the COVID-19 pandemic has brought drastic changes in various aspects, wide and deep, more significantly 
in the processes of HEIs. A variety of relevant issues emerged, and crucial attempts have been posed in the current literature. For instance, Daniel [46] provided some pragmatic guidance to the government, HEIs, and teachers in managing the educational consequences of COVID-19. Shahzad et al. [47] proposed a framework for identifying the success of e-learning portals by exploring the impact of information quality, system quality, and service quality on user satisfaction and the e-learning system used on the e-learning portal success. Mishra et al. [48] highlighted the online teaching-learning process perception of faculty and students during the COVID-19 pandemic and provided a roadmap on how HEIs can shift to online (i.e., distance) education with their existing resources. Other COVID-19 works with HEIs include online teaching strategies [4, 47, 48], e-learning [49-51], students' perceptions [52-54], challenges and opportunities [2, 55-57], responses [58-63], digital readiness [64, 65], online assessment $[66,67]$, and digital transformation [7, 68, 69]. This list is highly dynamic with the dramatic evolution of insights in promoting a good perspective in various areas of the education sector.

Although not well highlighted in the domain literature, the impact of the sudden and massive shift to e-learning on academics, particularly in developing economies, is significant in the continuum of the teaching-learning process. For instance, they lack the necessary training and technical infrastructure to facilitate online classes, required lesson plan design, learning materials (e.g., audio-visual and modules), and technical support groups [4]. Academics are also concerned with the lack of online teaching training and experience, slow Internet speed, WIFI coverage, design of the interface, content quality, system use and adoption, and technical infrastructure in remote areas, among others $[47,70]$. Furthermore, local culture and practices, skills and competencies, individual values, and attitudes are deemed to be crucial barriers to distance education [71]. Understanding these factors affecting e-learning becomes necessary to provide a better perspective on fine-tuning response initiatives to this sudden shift [1]. Thus, it is important for HEIs to continually motivate academics, either externally or intrinsically, cognitively, affectively, positively, or negatively, to sustain distance education or e-learning [12].

Emerging reports on e-learning acceptance modeling amidst COVID-19 can be observed in the domain literature. Sukendro et al. [50] explored the factors of predicting e-learning acceptance among sports science education students in HEIs in Indonesia using an extended TAM (i.e., the inclusion of facilitating condition as an external factor). Still, in one university in Indonesia, Mailizar et al. [28] adopted the TAM with system quality and e-learning experience in modeling factors of students' behavioral intention to use e-learning. In addition to the traditional constructs of TAM, Siron et al. [72] integrated other constructs such as student experiences, perceived enjoyment, computer anxiety, and perceived self-efficacy to examine the use of e-learning of a university in Indonesia. On the other hand, an updated version of the popular information system success model of Delone and McLean [73] was adopted by
Shahzad et al. [47] in modeling the e-learning portal acceptance of universities in Malaysia. In an Indian context, Saxena et al. [74] espoused e-learning quality as a predictor of learner satisfaction and adopted the SERVQUAL model to explore those factors that predict the quality of e-learning. In the case study of South Korea, Baber [75] extended TAM by incorporating instructor characteristics and student characteristics to evaluate e-learning adoption among management students at both the undergraduate and graduate levels. Abbad [76] reported a case in a university in Jordan and adopted the UTAUT to analyze students' intention of using an e-learning system (i.e., Moodle). Note that this list is not intended to be comprehensive due to the increasing attention scholars are paying to the domain field. At this point, it is noteworthy that (1) the majority of the works implemented SEM in analyzing acceptance models, and (2) limited attention is allotted to the perspective of academics in e-learning acceptance modeling, except for a few works (e.g., [77]).

However, the presence of individual-level values has not been explored in the current nexus of e-learning and the COVID-19 pandemic. These values are crucial to investigate the differences in acceptance modeling among different cultures [78]. Incorporating them into the context of e-learning is insightful as e-learning acceptance is associated with social factors and culture [16]. In this regard, Mehta et al. [16] developed integration of Schwartz's Theory of Human Values and UTAUT2 and introduced the VETA model. The VETA model is strongly inspired by some previous insights. For instance, Aparicio et al. [18] observed that the individualistic or collectivistic culture of students moderates perceived satisfaction on their assessment of individual impacts, with a consequence on organizational impacts. Tarhini et al. [79] found that subjective norms and quality of work-life are positively associated with the behavioral intention of students to adopt e-learning. On a follow-up work, Tarhini et al. [19] maintained the integration of subjective norms and quality of work-life as additional constructs for an extended TAM while exploring the moderating effects of cultural variables (i.e., masculinity/ femininity, individualism/collectivism, power distance, and uncertainty avoidance), measured at the individual level. They suggest that subjective norms and quality of work are sensitive to the differences in individual-cultural values. The formal integration of individual values was put forward by Mehta et al. [16] by highlighting that an argument explaining individual-level constructs (i.e., values) by way of grouplevel constructs (i.e., culture) would result in ecological fallacy.

Some insights into the VETA model have been explored to model the acceptance of e-learning during the COVID-19 pandemic. For instance, Jameel et al. [24] studied the effect of the constructs of UTAUT2 on the behavioral intention of Iraqi students in using e-learning. They found that performance expectancy, effort expectancy, facilitating conditions, and habit positively affect behavioral intention. In the case of the EU, Sitar-Tăut [27] explored a new model based on the UTAUT with hedonic motivation acting as a mediator as opposed to being an exogenous variable in the original 
structure of the UTAUT. They underscore the strong relationship between hedonic motivation and behavioral intention and between perceived effectiveness and hedonic motivation. Sitar-Tăut and Mican [25] developed an extension of the UTAUT to model the relationships between the constructs and personal innovativeness and information quality, with hedonic motivation and learning value as mediators. Their findings suggest the significant relationship between performance expectancy and hedonic motivation and the positive effect of hedonic motivation on behavioral intention to use. Fatimah et al. [26] proposed a model associating extroversion/introversion character (i.e., personal) qualities and collectivism/individualism (i.e., cultural) convictions on collaborative learning. Their work shows that the personality and cultural beliefs of students are associated with computer-supported collaborative learning. While these works offer some insights, the following gaps are deemed to be relevant: (1) the perspectives of academics in e-learning acceptance models were generally set aside despite their importance in the teaching-learning continuum, (2) the use of SEM in most studies has various drawbacks as accounted by Wei et al. [29] and Jeng and Tzeng [30], (3) as a consequence of using SEM, priority constructs were not determined which may be input to strategy formulation, and (4) the application of the constructs of the VETA model in evaluating e-learning acceptance of academics during the COVID-19 pandemic.

\section{Preliminaries of the DEMATEL Method}

Developed by the Battelle Memorial Institute of Geneva for a Science and Human Affairs Program in the 1970s, the DEMATEL method is a tool based on graph theory that considers a system as a graph, where elements or concepts and the causal relationships between these elements are represented as vertices and edges, respectively. It achieves two objectives: (1) to determine the total causal relationships among elements based on direct and indirect relations and (2) to categorize these elements into the net cause and net effect groups. In the DEMATEL, elements take on both cause and effect roles, and the final categorization identifies each element as a superior role, either cause or effect. These objectives of the DEMATEL enable a better understanding of the elements or concepts under consideration, which are often intertwined in convoluted problems [31, 32].

The computational algorithm of the DEMATEL is briefly described in the following steps. Note that the notations adopted in this work were lifted from Ocampo and Yamagishi [45]:

(1) Establish a system of a finite number of $n$ elements. Let $p_{1}, p_{2}, \ldots, p_{n}$ represent these $n$ elements.

(2) Generate the direct-relation matrix. An expert group of $H=1,2, \ldots, \mathbb{N}$ members elicits judgments on the causal influence of element $p_{i}$ on element $p_{j}$, $i, j \in\{1, \ldots, n\}$. This generates a set of $k$ direct-relation matrices $Z^{k}=\left(z_{i j}^{k}\right)_{n \times n}, k=1,2, \ldots, H$. Here, $x_{i j}$ represents such a causal influence, with an evaluation scale of $0,1,2,3$, and 4 , representing "no influence," "low influence," "medium influence," "high influence," and "very high influence," respectively. The aggregate direct-relation matrix $Z$, $\forall Z^{k}, k=1,2, \ldots, H$, is obtained by any predefined aggregation method (e.g., arithmetic mean method).

(3) Obtain the normalized direct-relation matrix. The following equation is used to produce such a matrix:

$$
G=\frac{z}{\max _{1 \leq i \leq n} \sum_{j=1}^{n} z_{i j}} .
$$

(4) Calculate the total relation matrix $T$, which represents all the direct and indirect causal relationships among the elements of the system. The total relation matrix $T=\left(t_{i j}\right)_{n \times n}$ is computed using

$$
T=G+G^{2}+G^{3}+\cdots=G(I-G)^{-1} \text {. }
$$

(5) Compute for the prominence and relation vectors. On the basis of equation (2), vectors $D$ and $R$ are obtained using the following equations, respectively:

$$
\begin{aligned}
& D=\left(\sum_{j=1}^{n} t_{i j}\right)_{n \times 1}=\left(t_{i}\right)_{n \times 1}, \\
& R=\left(\sum_{i=1}^{n} t_{i j}\right)_{1 \times n}=\left(t_{j}\right)_{1 \times n} .
\end{aligned}
$$

The $\left(D+R^{T}\right)$ vector (i.e., also known as the prominence vector) represents the relative importance of each element. Elements having greater values of $\left(D+R^{T}\right)$ indicate stronger relationships with other elements [44]. Those elements in the $\left(D-R^{T}\right)$ (i.e., also known as the relation vector) having $t_{i}-t_{j}>0, i=j$ belong to the net cause group, while those elements with $t_{i}-t_{j}<0, i=j$ belong to the net effect group. Elements in the net cause group are referred to as dispatchers, while those in the net effect group are receivers.

(6) Construct the prominence-relation map. This map shows the $\left(D+R^{T}, D-R^{T}\right)$ mapping of the elements. The directed relationships in the prominencerelation map are characterized by $t_{i j}$. However, some of these total relationships are insignificant, either in theory or in practice. To filter out these insignificant relations, a threshold value $\lambda$ is set in such a way that when $t_{i j} \geq \lambda$, then a directed edge from element $p_{i}$ to element $p_{j}$ is drawn in the prominence-relation map.

\section{Methodology}

4.1. Research Participants. An expert group of five academics with extensive background and experience in e-learning elicits judgments on the contextual relationships among constructs. All of them hold Ph.D. degrees. They are all working in universities in the Philippines, ranging from Assistant Professor to Full Professor positions. The average length of experience holding academic positions is 17 years, 
with an average of 11.6 years in supervisory and administrative functions. These academics have been working on a work-from-home arrangement since the start of the COVID-19 pandemic, engaging in the use of online learning and flexible learning systems. Also, they are involved in research in various areas (e.g., education, technology management, business management, information and communication technology, guidance, and counseling), having a range of 10 to 100 Scopus-indexed publications.

4.2. Research Instrument. The proposed constructs that best describe the integration of values in e-learning during the COVID-19 pandemic were obtained from Mehta et al. [16]. In summary, Table 1 presents the nine constructs, along with the codes for the brevity of presentation and corresponding brief descriptions. These include perceived performance expectancy (PE), effort expectancy (EE), behavioral intention (BI), social influence (SI), hedonic motivation (HM), price value (PV), habit (HAB), security (SE), tradition (TR), conformity $(\mathrm{CO})$, achievement $(\mathrm{AC})$, power (PO), and hedonism (HE).

From these constructs, a questionnaire was structured in a way that allows the research participants to evaluate the perceived degree of casual relation from one construct to another construct. Each question contains two parts: (1) evaluating whether one construct causes another construct and (2) if the answer in (1) is Yes, rating the degree of causal impact using the scale described in Table 2. A sample question becomes "Does Performance expectancy cause the existence of Effort expectancy? If Yes, by how much?" The questionnaire contains 144 questions of this type. The responses would form the initial direct-relation matrix from a participant within the context of the DEMATEL. With a purposive sampling technique, each questionnaire was sent via e-mail to an invited participant, following the qualifications set in identifying the composition of the expert group. Inquiries from the participants regarding the questionnaire were promptly addressed.

4.3. Proposed Research Design. The proposed approach for modeling the constructs of the recently established VETA model in e-learning acceptance is described in the following:

Step 1: identify the constructs that comprise the model. The constructs presented in Table 1 obtained from Mehta et al. [16] comprise the elements of the model (i.e., vertices or nodes of a directed graph).

Step 2: set up the initial direct-relation matrices in linguistic variables. With the rating scale shown in Table 2, the questions in the questionnaire would form the initial direct-relation matrix; each corresponds to the decision-maker $k=1,2, \ldots, H$.

Step 3: generate the initial direct-relation matrices in numerical values. Substituting the linguistic variables with their corresponding equivalent scores in Table 2 produces the initial direct-relation matrices.
Step 4: aggregate the initial direct-relation matrices. The initial direct-relation matrices, each corresponding to a participant, are combined to generate an aggregate initial direct-relation matrix.

Step 5: calculate the normalized direct-relation matrix. Applying equation (1) to the aggregate initial directrelation matrix, the normalized direct-relation matrix is obtained.

Step 6: obtain the total relation matrix and the prominence and relation vectors. The total relation matrix is generated through equation (2). On this basis, the prominence and relation vectors are calculated based on equations (3) and (4).

Step 7: obtain the adjacency matrix. The threshold value $\lambda$ is set for the significant $t_{i j}$ values of the total relation matrix. If $t_{i j} \geq \lambda$, then construct $i$ is adjacent to construct $j$; that is, the $(i, j)$ position of the adjacency matrix is equal to one. Otherwise, its value is zero.

Step 8: construct the prominence-relation map. By plotting the $\left(D+R^{T}, D-R^{T}\right)$ points that correspond to all constructs and taking the adjacency matrix into consideration, the prominence-relation map is constructed as a directed graph.

\section{Results and Discussion}

In this section, we detail the results of the corresponding procedural steps in Section 4.3. For tractability, the relevant results consequent to the implementation of the DEMATEL method are presented here. A sample initial direct-relation matrix in linguistic variables from an expert is shown in Table 3. Using Table 1, the initial direct-relation matrices were obtained. Table 4 presents a sample that is an equivalent matrix to Table 3 . The aggregate initial direct-relation matrix was obtained using an arithmetic mean method, an approach widely adopted in the domain literature (e.g., [80]). Table 5 shows the aggregate initial direction-relation matrix. Applying equation (1) to Table 5, the normalized directrelation matrix was obtained, as shown in Table 6. Finally, the total relation matrix was generated using equation (2). From the total relation matrix, the prominence and relation vectors were obtained from equations (3) and (4). They are all presented in Table 7 . The threshold value $\lambda$ is obtained by computing for the 75th percentile of all $t_{i j}$ values in Table 7 . This choice is consistent with other DEMATEL-based studies. It yields $\lambda=0.021$. Table 8 shows the adjacency matrix. Combining the information obtained from prominence and relation vectors reflected in Table 7 and the adjacency matrix in Table 8, Figure 1 illustrates the prominence-relation map.

Results show that effort expectancy (EE), hedonic motivation (HM), price value (PV), habit (HAB), security (SE), tradition (TR), conformity (CO), achievement (AC), power (PO), and hedonism (HE) belong to the net cause group, which implies that they are deemed to be the primary motivating constructs in e-learning considering individual values of users. Attaining them is crucial to the successful implementation of e-learning during the COVID-19 
TABLE 1: Constructs on integrating individual values in e-learning adoption.

\begin{tabular}{|c|c|c|}
\hline Code & Construct & Description \\
\hline $\mathrm{PE}$ & $\begin{array}{l}\text { Performance } \\
\text { expectancy }\end{array}$ & The degree to which an individual believes that system use will yield gain in work performance \\
\hline $\mathrm{EE}$ & Effort expectancy & The degree to which system use is free from effort \\
\hline BI & Behavioral intention & The strength of one's intention to perform a specified behavior \\
\hline SI & Social influence & The perception of group influence on an individual's decision \\
\hline HM & Hedonic motivation & $\begin{array}{c}\text { Linked to learner enjoyment, playfulness with e-learning, learning strategy, the flow of the learning } \\
\text { experience, and engagement, as well as the success of online learning }\end{array}$ \\
\hline PV & Price value & $\begin{array}{l}\text { Linked to a user making a cost-benefit decision, where technologies are more useful if the benefits are more } \\
\text { significant than the cost incurred }\end{array}$ \\
\hline HAB & Habit & Established routines in the workplace for find \\
\hline SE & Security & $\begin{array}{c}\text { Associated with personal security (personal health, safety, and wellbeing) and societal security (stable social } \\
\text { order) }\end{array}$ \\
\hline TR & Tradition & Indicating an individual's priority on existing paradigms within their organization \\
\hline $\mathrm{CO}$ & Conformity & $\begin{array}{c}\text { The degree to which the rules laid down by the organization in terms of mandatory or endorsed training } \\
\text { programs are followed }\end{array}$ \\
\hline $\mathrm{AC}$ & Achievement & $\begin{array}{c}\text { Including the demonstration of competence by the standards of one's reference groups and the pursuit of } \\
\text { personal success }\end{array}$ \\
\hline $\mathrm{PO}$ & Power & $\begin{array}{c}\text { Associated with dominance over resources and people are linked to both formal and informal status in an } \\
\text { organization }\end{array}$ \\
\hline $\mathrm{HE}$ & Hedonism & Incorporating intrinsic motivation, including novelty, challenge, excitement, and pleasure \\
\hline
\end{tabular}

TABLE 2: The linguistic rating scale for DEMATEL.

\begin{tabular}{lcc}
\hline Linguistic variables & Code & Influence score \\
\hline No influence & NI & 0 \\
Low influence & LI & 1 \\
Medium influence & MI & 2 \\
High influence & HI & 3 \\
Very high influence & VHI & 4 \\
\hline
\end{tabular}

TABLE 3: Sample initial direct-relation matrix in linguistic variables.

\begin{tabular}{|c|c|c|c|c|c|c|c|c|c|c|c|c|c|}
\hline & $\mathrm{PE}$ & $\mathrm{EE}$ & BI & SI & HM & PV & HAB & SE & TR & $\mathrm{CO}$ & AC & $\mathrm{PO}$ & $\mathrm{HE}$ \\
\hline $\mathrm{PE}$ & & & HI & & & & & & & LI & & & \\
\hline $\mathrm{EE}$ & & & HI & & & & & & & & & & \\
\hline BI & & & & & & & & & & & & & \\
\hline SI & HI & & LI & & & & & & & & & & \\
\hline $\mathrm{HM}$ & HI & & MI & & & & & & & & & & \\
\hline PV & MI & & LI & & & & & & & & & & \\
\hline HAB & & & HI & & & & & & & & & & \\
\hline SE & MI & & & LI & & & & & & & & & \\
\hline TR & HI & & & HI & & & & & & & & & \\
\hline $\mathrm{CO}$ & HI & & & HI & & & & & & & & & \\
\hline AC & HI & & & $\mathrm{HI}$ & & HI & & & & & & & \\
\hline PO & LI & & & $\mathrm{HI}$ & & MI & & & & & & & \\
\hline $\mathrm{HE}$ & & & & & HI & & & & & & & & \\
\hline
\end{tabular}

pandemic with the presence of various values-related features. These constructs in the net cause group have a more influential impact $(D)$ than influenced impact $(R)$. On the other hand, performance expectancy $(\mathrm{PE})$, behavioral intention (BI), and social influence (SI) are in the net effect group. They tend to be influenced by the constructs in the net cause group as their $\left(D-R^{T}\right)$ values are negative, which implies that the influential impact $(D)$ of these constructs is lower than their influenced impact $(R)$. For instance, PE is influenced by all constructs except EE, BI, and HAB. This finding is similar to SI with the addition of PV. On the other hand, BI is impacted by all constructs. These relationships are appropriately depicted in Table 1, where most edges are directed to these PE, BI, and SI.

We compare these observations with the findings of Mehta et al. [16]. At the outset, it should be noted that the model introduced by Mehta et al. [16] is hypothesized by exploring evidence from previous studies. However, in this work, participants are allowed to perceive the causal relation of one construct on another construct while setting aside rigorous theoretical support. The process then becomes exploratory, and the results must be used for future 
TABLE 4: Sample initial direct-relation matrix in numerical values.

\begin{tabular}{|c|c|c|c|c|c|c|c|c|c|c|c|c|c|}
\hline & $\mathrm{PE}$ & $\mathrm{EE}$ & BI & SI & HM & PV & HAB & SE & TR & $\mathrm{CO}$ & $\mathrm{AC}$ & $\mathrm{PO}$ & $\mathrm{HE}$ \\
\hline $\mathrm{PE}$ & & & 3 & & & & & & & 1 & & & \\
\hline $\mathrm{EE}$ & & & 3 & & & & & & & & & & \\
\hline BI & & & & & & & & & & & & & \\
\hline SI & 3 & & 1 & & & & & & & & & & \\
\hline $\mathrm{HM}$ & 3 & & 2 & & & & & & & & & & \\
\hline PV & 2 & & 1 & & & & & & & & & & \\
\hline HAB & & & 3 & & & & & & & & & & \\
\hline SE & 2 & & & 1 & & & & & & & & & \\
\hline $\mathrm{TR}$ & 3 & & & 3 & & & & & & & & & \\
\hline $\mathrm{CO}$ & 3 & & & 3 & & & & & & & & & \\
\hline $\mathrm{AC}$ & 3 & & & 3 & & 3 & & & & & & & \\
\hline $\mathrm{PO}$ & 1 & & & 3 & & 2 & & & & & & & \\
\hline $\mathrm{HE}$ & & & & & 3 & & & & & & & & \\
\hline
\end{tabular}

TABle 5: Aggregate initial direct-relation matrix.

\begin{tabular}{lccccccccccccc}
\hline & PE & EE & BI & SI & HM & PV & HAB & SE & TR & CO & AC & PO & HE \\
\hline PE & 0 & 0 & 3.2 & 0 & 0 & 0 & 0 & 0 & 0 & 0.2 & 1.2 & 0 & 0 \\
EE & 0 & 0 & 3.4 & 0 & 0 & 0 & 0 & 0 & 0 & 0 & 0 & 0 & 0 \\
BI & 0 & 0 & 0 & 0 & 0 & 0 & 0.2 & 0 & 0 & 0 & 0 & 0 & 0 \\
SI & 3.2 & 0 & 2.6 & 0 & 0 & 0 & 0 & 0 & 0 & 0 & 0 & 0 & 0 \\
HM & 3.4 & 0 & 2.6 & 0 & 0 & 0 & 0 & 0 & 0 & 0 & 0 & 0 & 0 \\
PV & 3.2 & 0 & 2.8 & 0 & 0 & 0 & 0 & 0 & 0 & 0 & 0 & 0 & 0 \\
HAB & 0 & 0 & 3.2 & 0 & 0 & 0 & 0 & 0 & 0 & 0 & 0 & 0 & 0 \\
SE & 2.4 & 0 & 0 & 2.2 & 0 & 0 & 0 & 0 & 0 & 0 & 0 & 0 \\
TR & 2.8 & 0 & 0 & 3.2 & 0 & 0 & 0 & 0 & 0 & 0 & 0 & 0 \\
CO & 2.8 & 0 & 0 & 2.4 & 0 & 0 & 0 & 0 & 0 & 0 & 0 & 0 \\
AC & 3.2 & 0 & 0 & 3.2 & 0 & 3.2 & 0 & 0 & 0 & 0 & 0 & 0 \\
PO & 2.4 & 0 & 0 & 2.8 & 0 & 2.4 & 0 & 0 & 0 & 0 & 0 & 0 \\
HE & 0 & 0 & 0 & 0 & 2.4 & 0 & 0 & 0 & 0 & 0 & 0 & 0 \\
\hline
\end{tabular}

TABLE 6: Normalized direct-relation matrix.

\begin{tabular}{|c|c|c|c|c|c|c|c|c|c|c|c|c|c|}
\hline & $\mathrm{PE}$ & $\mathrm{EE}$ & BI & SI & $\mathrm{HM}$ & PV & HAB & SE & TR & $\mathrm{CO}$ & $\mathrm{AC}$ & $\mathrm{PO}$ & $\mathrm{HE}$ \\
\hline PE & 0 & 0 & 0.333 & 0 & 0 & 0 & 0 & 0 & 0 & 0.021 & 0.125 & 0 & 0 \\
\hline $\mathrm{EE}$ & 0 & 0 & 0.354 & 0 & 0 & 0 & 0 & 0 & 0 & 0 & 0 & 0 & 0 \\
\hline BI & 0 & 0 & 0 & 0 & 0 & 0 & 0.021 & 0 & 0 & 0 & 0 & 0 & 0 \\
\hline SI & 0.333 & 0 & 0.271 & 0 & 0 & 0 & 0 & 0 & 0 & 0 & 0 & 0 & 0 \\
\hline $\mathrm{HM}$ & 0.354 & 0 & 0.271 & 0 & 0 & 0 & 0 & 0 & 0 & 0 & 0 & 0 & 0 \\
\hline PV & 0.333 & 0 & 0.292 & 0 & 0 & 0 & 0 & 0 & 0 & 0 & 0 & 0 & 0 \\
\hline HAB & 0 & 0 & 0.333 & 0 & 0 & 0 & 0 & 0 & 0 & 0 & 0 & 0 & 0 \\
\hline SE & 0.250 & 0 & 0 & 0.229 & 0 & 0 & 0 & 0 & 0 & 0 & 0 & 0 & 0 \\
\hline TR & 0.292 & 0 & 0 & 0.333 & 0 & 0 & 0 & 0 & 0 & 0 & 0 & 0 & 0 \\
\hline $\mathrm{CO}$ & 0.292 & 0 & 0 & 0.250 & 0 & 0 & 0 & 0 & 0 & 0 & 0 & 0 & 0 \\
\hline $\mathrm{AC}$ & 0.333 & 0 & 0 & 0.333 & 0 & 0.333 & 0 & 0 & 0 & 0 & 0 & 0 & 0 \\
\hline $\mathrm{PO}$ & 0.250 & 0 & 0 & 0.292 & 0 & 0.250 & 0 & 0 & 0 & 0 & 0 & 0 & 0 \\
\hline $\mathrm{HE}$ & 0 & 0 & 0 & 0 & 0.250 & 0 & 0 & 0 & 0 & 0 & 0 & 0 & 0 \\
\hline
\end{tabular}

validation. Nevertheless, their perception is based on knowledge and experience, which may reveal important points of discussion. The findings of Mehta et al. [16] suggest that $\mathrm{BI}$ is only directly influenced by $\mathrm{PV}, \mathrm{PE}, \mathrm{EE}$, and $\mathrm{HAB}$ in one or two cases under consideration. However, their validated model shows that, under both direct and indirect causal relationships, all except SE and PO influence behavioral intention. The notion of the total influence of the DEMATEL (i.e., as shown in equation (2)), with the help of the threshold value, captures these relationships in Figure 1.
Under a pandemic, given possible exposure to COVID-19 associated with mobility, public transport, and face-to-face classes, it is straightforward that personal and societal security via public health always favors the use of e-learning platforms (i.e., satisfying SE). On the other hand, with the national measure of banning face-to-face classes, consequently, processes within HEIs are compelled to shift online. As a result, power over people and resources is maintained with the adoption of e-learning platforms; thus, personal inclination to hold power in an organization such as an HEI 
TABLE 7: Total relation matrix with prominence and relation vectors.

\begin{tabular}{lcccccccccccccccccccccc}
\hline & PE & EE & BI & SI & HM & PV & HAB & SE & TR & CO & AC & PO & HE & D & R & D + R & D - R & Cluster \\
\hline PE & 0.084 & 0 & 0.391 & 0.051 & 0 & 0.045 & 0.008 & 0 & 0 & 0.023 & 0.135 & 0 & 0 & 0.737 & 3.551 & 4.288 & -2.815 & Net effect \\
EE & 0 & 0 & 0.357 & 0 & 0 & 0 & 0.007 & 0 & 0 & 0 & 0 & 0 & 0 & 0.364 & 0.000 & 0.364 & 0.364 & Net cause \\
BI & 0 & 0 & 0.007 & 0 & 0 & 0 & 0.021 & 0 & 0 & 0 & 0 & 0 & 0 & 0.028 & 3.812 & 3.840 & -3.784 & Net effect \\
SI & 0.361 & 0 & 0.403 & 0.017 & 0 & 0.015 & 0.008 & 0 & 0 & 0.008 & 0.045 & 0 & 0 & 0.857 & 1.651 & 2.508 & -0.794 & Net effect \\
HM & 0.384 & 0 & 0.411 & 0.018 & 0 & 0.016 & 0.009 & 0 & 0 & 0.008 & 0.048 & 0 & 0 & 0.894 & 0.250 & 1.144 & 0.644 & Net cause \\
PV & 0.361 & 0 & 0.424 & 0.017 & 0 & 0.015 & 0.009 & 0 & 0 & 0.008 & 0.045 & 0 & 0 & 0.879 & 0.773 & 1.652 & 0.106 & Net cause \\
HAB & 0 & 0 & 0.336 & 0 & 0 & 0 & 0.007 & 0 & 0 & 0 & 0 & 0 & 0 & 0.343 & 0.100 & 0.443 & 0.242 & Net cause \\
SE & 0.354 & 0 & 0.190 & 0.246 & 0 & 0.015 & 0.004 & 0 & 0 & 0.007 & 0.044 & 0 & 0 & 0.860 & 0.000 & 0.860 & 0.860 & Net cause \\
TR & 0.437 & 0 & 0.248 & 0.354 & 0 & 0.018 & 0.005 & 0 & 0 & 0.009 & 0.055 & 0 & 0 & 1.126 & 0.000 & 1.126 & 1.126 & Net cause \\
CO & 0.406 & 0 & 0.215 & 0.269 & 0 & 0.017 & 0.004 & 0 & 0 & 0.008 & 0.051 & 0 & 0 & 0.971 & 0.095 & 1.066 & 0.876 & Net cause \\
AC & 0.602 & 0 & 0.406 & 0.362 & 0 & 0.358 & 0.008 & 0 & 0 & 0.013 & 0.075 & 0 & 0 & 1.824 & 0.569 & 2.393 & 1.255 & Net cause \\
PO & 0.467 & 0 & 0.321 & 0.314 & 0 & 0.269 & 0.007 & 0 & 0 & 0.010 & 0.058 & 0 & 0 & 1.446 & 0.000 & 1.446 & 1.446 & Net cause \\
HE & 0.096 & 0 & 0.103 & 0.004 & 0.250 & 0.004 & 0.002 & 0 & 0 & 0.002 & 0.012 & 0 & 0 & 0.473 & 0.000 & 0.473 & 0.473 & Net cause \\
\hline
\end{tabular}

Table 8: Adjacency matrix.

\begin{tabular}{lccccccccccccc}
\hline & PE & EE & BI & SI & HM & PV & HAB & SE & TR & CO & AC & PO & HE \\
\hline PE & 1 & 0 & 1 & 1 & 0 & 1 & 0 & 0 & 0 & 1 & 1 & 0 & 0 \\
EE & 0 & 0 & 1 & 0 & 0 & 0 & 0 & 0 & 0 & 0 & 0 & 0 & 0 \\
BI & 0 & 0 & 0 & 0 & 0 & 0 & 1 & 0 & 0 & 0 & 0 & 0 & 0 \\
SI & 1 & 0 & 1 & 0 & 0 & 0 & 0 & 0 & 0 & 0 & 1 & 0 \\
HM & 1 & 0 & 1 & 0 & 0 & 0 & 0 & 0 & 0 & 0 & 1 & 0 \\
PV & 1 & 0 & 1 & 0 & 0 & 0 & 0 & 0 & 0 & 0 & 1 & 0 \\
HAB & 0 & 0 & 1 & 0 & 0 & 0 & 0 & 0 & 0 & 0 & 0 & 0 \\
SE & 1 & 0 & 1 & 1 & 0 & 0 & 0 & 0 & 0 & 0 & 1 & 0 \\
TR & 1 & 0 & 1 & 1 & 0 & 0 & 0 & 0 & 0 & 0 & 1 & 0 \\
CO & 1 & 0 & 1 & 1 & 0 & 0 & 0 & 0 & 0 & 0 & 1 & 0 \\
AC & 1 & 0 & 1 & 1 & 0 & 1 & 0 & 0 & 0 & 0 & 1 & 0 \\
PO & 1 & 0 & 1 & 1 & 0 & 1 & 0 & 0 & 0 & 0 & 1 & 0 \\
HE & 1 & 0 & 1 & 0 & 1 & 0 & 0 & 0 & 0 & 0 & 0 & 0 \\
\hline
\end{tabular}

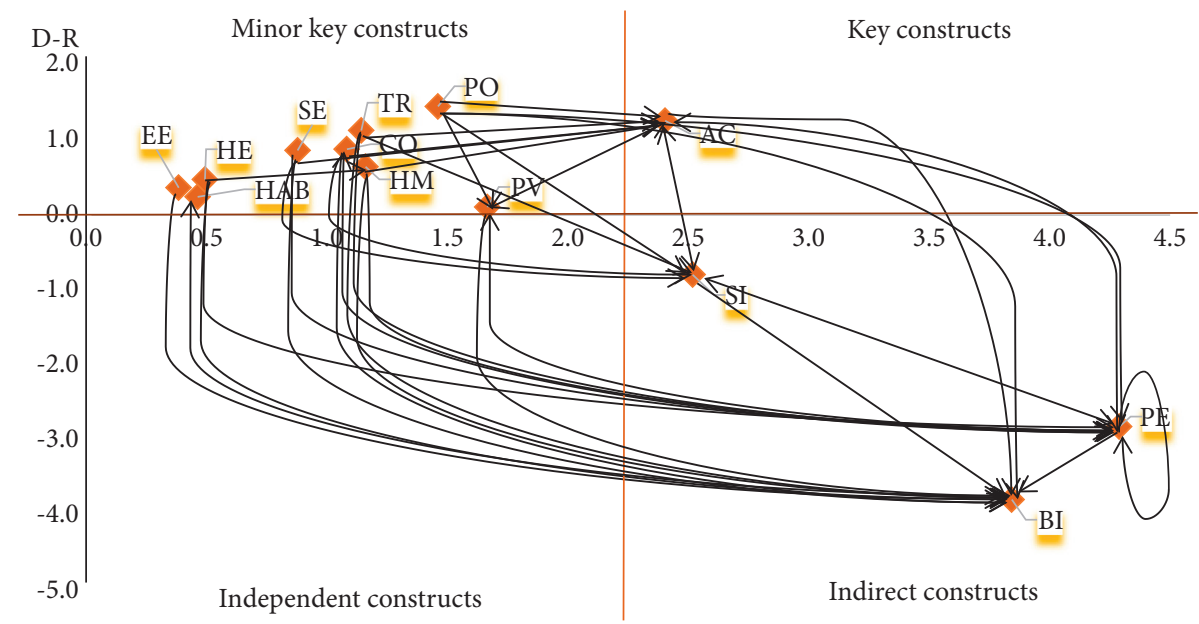

Figure 1: The prominence-relation map.

would inspire academics for e-learning. While the motivations behind the other constructs influencing BI are not discussed here following the findings of Mehta et al. [16], the conditions under the COVID-19 pandemic justify the causal relationships between SE and PO on $\mathrm{BI}$.

In the PE construct, Mehta et al. [16] found that CO, TR, $\mathrm{AC}, \mathrm{HE}, \mathrm{HM}, \mathrm{SI}, \mathrm{PV}$, and EE have either direct or indirect influence on PE. These relationships are supported by the findings of this work, except for EE. Currently, since all courses are offered under e-learning platforms with no option for physical meetings, laboratory courses would require academics to exert more effort in e-learning than in face-to-face classes. Depending on the topic within a given course, the degree of effort hugely varies. Thus, this finding is 
not supported by the findings of the DEMATEL. On the other hand, the influence of SE and PO on PE is supported in this work, in contrast to Mehta et al. [16]. With the public health dilemma, an individual's sense of personal and societal security associated with e-learning adoption would improve work performance. Academics are better off using e-learning than the anxiety they might experience in and out of the workplace while holding physical classes. Also, the power that academics would gain from e-learning improves work performance, as current yardsticks of academic performance rely on online activities. Again, these conditions under the COVID-19 pandemic support the influence of SE and $\mathrm{PO}$ on $\mathrm{PE}$.

The findings of Mehta et al. [16] also suggest that TR, AC, and CO support SI. These are both supported in Figure 1. Aside from these relationships, the DEMATEL approach considers the causal impacts of PE, SE, and PO on SI. These insights may be explained as follows: The belief that jobrelated goals are achieved and that performance is improved through e-learning fosters others to adopt the same. This reasoning can be extended to $\mathrm{SE}$ and PO. Perception of enhanced security and power when using e-learning would attract others to do the same. These links are enhanced under a pandemic where other modes of teaching and learning are almost nonexistent. Consistent with Mehta et al. [16], hedonism influences hedonic motivation, and the explanation is relatively straightforward. The findings of this work also support the influence of $\mathrm{AC}$ on PV. In addition, PE and PO are also linked to PV, which are not reflected in Mehta et al. [16]. One striking set of findings described in this work is the causal influence of PE, SI, HM, PV, SE, TR, CO, and PO on AC, which was not hypothesized by the VETA model of Mehta et al. [16]. This finding implies that these constructs promote the belief of self-achievement among academics. However, it must be noted that while in-degrees of this node (or construct) are many, which may suggest $\mathrm{AC}$ as a net effect construct, AC remains a net cause. Thus, confirmatory studies must be implemented to evaluate these relationships. Nevertheless, these insights may pave crucial points of discussion.

Ultimately identifying the critical constructs must simultaneously consider both $\left(D+R^{T}\right)$ and $\left(D-R^{T}\right)$ vectors. In achieving this, we refer to Figure 1 and categorize all constructs into four distinct categories: minor key constructs (low prominence and high relation), key constructs (high prominence and high relation), indirect constructs (high prominence and low relation), and independent constructs (low prominence and low relation). Based on Figure 1, the minor key construct comprises EE, HM, PV, HAB, SE, TR, $\mathrm{CO}, \mathrm{PO}$, and $\mathrm{HE}$. The key construct only includes achievement (AC). The indirect construct category is composed of performance expectancy (PE), behavioral intention (BI), and social influence (SI), which consequently belong to the net effect group. No independent construct is identified. Here, we would highlight the key construct category as it is crucial for policy-making, particularly in crafting initiatives to improve e-learning during the pandemic. This finding is also illustrated in the empirical finding of Mehta et al. [16], where achievement influences performance expectancy, social influence, and price value, leading to the behavioral intention of adopting e-learning. This indicates that the perception of academics on e-learning is linked to a sense of achievement. Thus, HEIs must concentrate their resources on investing in initiatives that would emphasize to academics the benefits of e-learning in achieving their goals. Investments in seminars, training, and workshops may be facilitated to improve such required knowledge. Such initiatives would enhance the use of e-learning among academics in HEIs.

\section{Conclusion and Future Work}

As the suspension of physical classes at schools is still an important measure in curbing COVID-19 cases, universities are compelled to instantly shift the teaching-learning process to digital platforms on a massive scale. Amplified by poor technical infrastructure, academic incompetency, and lack of resources, particularly in the developing economies, this sudden shift induces a stressful environment for academics in carrying out teaching duties, not to mention the other roles they have in their respective organizations. The current literature quickly catches up with reports on better understanding the various facets of education affected by the COVID-19 pandemic. An important highlight focuses on understanding the role of individual values in e-learning acceptance models. An important contribution of Mehta et al. [16], which offers a model based on 13 constructs, becomes the motivation of this work. Despite providing crucial insights on the problem domain, the work of Mehta et al. [16] with SEM as their mode of the analysis entails few drawbacks, which include (1) the limited perspectives of academics, (2) the serious shortcomings in the use of SEM in most studies, (3) identification of priority constructs for decision-making, and (4) the application of the constructs of the VETA model under the COVID-19 pandemic in a specific setting.

This work offers advances on these drawbacks by adopting the DEMATEL approach in modeling the constructs explaining the success of e-learning while integrating values in the advent of the COVID-19 pandemic. In this study, the causal relationships between the 13 constructs of the VETA model (i.e., performance expectancy, effort expectancy, behavioral intention, social influence, hedonic motivation, price value, habit, security, tradition, conformity, achievement, power, and hedonism) were evaluated. Results indicate that performance expectancy, behavioral intention, and social influence are the effects of effort expectancy, hedonic motivation, price value, habit, security, tradition, conformity, achievement, power, and hedonism constructs. Findings also reveal that some findings are consistent with Mehta et al. [16], while some differences are observed. The new insights into this work, albeit exploratory, may be associated with the casual loops and interdependencies among constructs that are not captured in SEM. Finally, consistent with the empirical evidence of Mehta et al. [16], the achievement construct drives academics to adopt e-learning. Initiatives that would better highlight the benefits of e-learning in self-achievement among academics would 
increase the use of e-learning. With these insights, this work reveals that DEMATEL has considerable potential in modeling studies in education and learning, which are scarce in the domain literature.

Nevertheless, the findings of this work must be treated with limitations. First, the limited number of experts may be a ground for future research. With an expanded number of experts, future works may explore the same model along with the proposed approach (i.e., DEMATEL) to test the validity of the findings. Second, the proposed approach could be used in any extended model, capturing some salient constructs on how academics adopt e-learning. Third, other DEMATEL extensions could be explored as a methodological extension to evaluate any acceptance models. Fourth, the use of different modeling techniques (e.g., system dynamics modeling and interpretative structural modeling) could also be used to evaluate the hypothesized model of Mehta et al. [16]. Fifth, since the findings contain idiosyncrasies, they must be interpreted with caution that they are exploratory in nature and empirical data is needed for validation. Finally, a resulting construct prioritization problem may be carried out in future research, along with the use of multiattribute decision-making techniques.

\section{Data Availability}

The survey data used to support the findings of this study are included within the article.

\section{Conflicts of Interest}

The authors declare that they have no conflicts of interest.

\section{References}

[1] M. Rizun and A. Strzelecki, "Students' acceptance of the COVID-19 impact on shifting higher education to distance learning in Poland," International Journal of Environmental Research and Public Health, vol. 17, no. 18, p. 6468, 2020.

[2] S. Donitsa-Schmidt and R. Ramot, "Opportunities and challenges: teacher education in Israel in the Covid-19 pandemic," Journal of Education for Teaching, vol. 46, no. 4, pp. 586-595, 2020.

[3] P. Sahu, "Closure of universities due to Coronavirus Disease 2019 (COVID-19): impact on education and mental health of students and academic staff," Cureus, vol. 12, no. 4, pp. e7541-e7546, 2020.

[4] W. Bao, "COVID-19 and online teaching in higher education: a case study of Peking University," Human Behavior and Emerging Technologies, vol. 2, no. 2, pp. 113-115, 2020.

[5] C. J. Thomas, "Coronavirus and challenging times for education in developing countries," Brookings, :http://D:/ COVID/Coronavirus\%20and\%20challenging\%20times\% 20 for\%20education\%20in\%20developing\%20countries.html, 2020.

[6] M. Teräs, J. Suoranta, H. Teräs, and M. Curcher, "Post-Covid19 education and education technology 'solutionism': a seller's market," Postdigital Science and Education, vol. 2, no. 3, pp. 863-878, 2020.

[7] D. Mhlanga and T. Moloi, "COVID-19 and the digital transformation of education: what are we learning on 4IR in South Africa?" Education Sciences, vol. 10, no. 7, p. 180, 2020.
[8] J. König, D. J. Jäger-Biela, and N. Glutsch, “Adapting to online teaching during COVID-19 school closure: teacher education and teacher competence effects among early career teachers in Germany," European Journal of Teacher Education, vol. 43, no. 4, pp. 608-622, 2020.

[9] Sangeeta and U. Tandon, "Factors influencing adoption of online teaching by school teachers: a study during COVID-19 pandemic," Journal of Public Affairs, 2020.

[10] N. T. T. Ho, S. Sivapalan, H. H. Pham, L. T. M. Nguyen, A. T. V. Pham, and H. V. Dinh, "Students' adoption of e-learning in emergency situation: the case of a Vietnamese university during COVID-19," Interactive Technology and Smart Education, 2020.

[11] R. S. Al-Maroof, S. A. Salloum, A. E. Hassanien, and K. Shaalan, "Fear from COVID-19 and technology adoption: the impact of Google Meet during Coronavirus pandemic," Interactive Learning Environments, pp. 1-16, 2020.

[12] I. O. Panisoara, I. Lazar, G. Panisoara, R. Chirca, and A. S. Ursu, "Motivation and continuance intention towards online instruction among teachers during the COVID-19 pandemic: the mediating effect of burnout and technostress," International Journal of Environmental Research and Public Health, vol. 17, no. 21, p. 8002, 2020.

[13] T. Abbas, "Social factors affecting students' acceptance of e-learning environments in developing and developed countries," Journal of Hospitality and Tourism Technology, vol. 7, no. 2, pp. 200-212, 2016.

[14] T.-H. Chu and Y.-Y. Chen, "With good we become good: understanding e-learning adoption by theory of planned behavior and group influences," Computers \& Education, vol. 92-93, pp. 37-52, 2016.

[15] G. Olasina, "Human and social factors affecting the decision of students to accept e-learning," Interactive Learning Environments, vol. 27, no. 3, pp. 363-376, 2019.

[16] A. Mehta, N. P. Morris, B. Swinnerton, and M. Homer, "The influence of values on e-learning adoption," Computers \& Education, vol. 141, Article ID 103617, 2019.

[17] Y. H. Lee, C. Hsiao, and S. H. Purnomo, "An empirical examination of individual and system characteristics on enhancing e-learning acceptance," Australasian Journal of Educational Technology, vol. 30, no. 5, 2014.

[18] M. Aparicio, F. Bacao, and T. Oliveira, "Cultural impacts on e-learning systems' success," The Internet and Higher Education, vol. 31, pp. 58-70, 2016.

[19] A. Tarhini, K. Hone, X. Liu, and T. Tarhini, "Examining the moderating effect of individual-level cultural values on users' acceptance of e-learning in developing countries: a structural equation modeling of an extended technology acceptance model," Interactive Learning Environments, vol. 25, no. 3, pp. 306-328, 2017.

[20] P. Ramírez-Correa, A. Mariano-Melo, and J. Alfaro-Pérez, "Predicting and explaining the acceptance of social video platforms for learning: the case of Brazilian youtube users," Sustainability, vol. 11, no. 24, p. 7115, 2019.

[21] R. Ambarwati, Y. D. Harja, Y. D. Harja, and S. Thamrin, "The role of facilitating conditions and user habits: a case of Indonesian online learning platform," The Journal of Asian Finance, Economics and Business, vol. 7, no. 10, pp. 481-489, 2020.

[22] Y. D. Harja, R. Ambarwati, and G. A. Handiwibowo, "A technology acceptance framework inside the Indonesian online learning platform," Journal of Physics: Conference Series, vol. 1783, no. 1, Article ID 012037, 2021. 
[23] P. Wannapiroon, N. Kaewrattanapat, and J. Premsmith, "Development of cloud learning management systems for higher education institutions," in Proceedings of the 2019 Research, Invention, and Innovation Congress (RI2C), pp. 1-6, IEEE, Bangkok, Thailand, December 2019.

[24] A. S. Jameel, S. N. Abdalla, and M. A. Karem, "November). Behavioural intention to use E-learning from student's perspective during COVID-19 pandemic," in Proceedings of the 2020 2nd Annual International Conference on Information and Sciences (AiCIS), pp. 165-171, IEEE, Fallujah, Iraq, November 2020.

[25] D. A. Sitar-Taut and D. Mican, "Mobile learning acceptance and use in higher education during social distancing circumstances: an expansion and customization of UTAUT2," Online Information Review, 2021.

[26] F. Fatimah, S. I. Rajiani, and E. W. Abbas, "Cultural and individual characteristics in adopting computer-supported collaborative learning during COVID-19 outbreak: willingness or obligatory to accept technology?" Management Science Letters, vol. 11, no. 2, pp. 373-378, 2021.

[27] D. A. Sitar-Tăut, "Mobile learning acceptance in social distancing during the COVID-19 outbreak: the mediation effect of hedonic motivation," Human Behavior and Emerging Technologies, vol. 3, no. 3, pp. 366-378, 2021.

[28] M. Mailizar, D. Burg, and S. Maulina, "Examining university students' behavioural intention to use e-learning during the COVID-19 pandemic: an extended TAM model," Education and Information Technologies, 2021.

[29] P. L. Wei, J. H. Huang, G. H. Tzeng, and S. I. Wu, "Causal modeling of web-advertising effects by improving SEM based on DEMATEL technique," International Journal of Information Technology and Decision Making, vol. 9, no. 5, pp. 799-829, 2010.

[30] D. J.-F. Jeng and G.-H. Tzeng, "Social influence on the use of clinical decision support systems: revisiting the unified theory of acceptance and use of technology by the fuzzy DEMATEL technique," Computers \& Industrial Engineering, vol. 62, no. 3, pp. 819-828, 2012.

[31] A. Gabus and E. Fontela, World Problems, an Invitation to Further Thought within the Framework of DEMATEL, Battelle Geneva Research Centre, Geneva, Switzerland, 1972.

[32] A. Gabus and E. Fontela, "Perceptions of the world problematic: communication procedure, communicating with those bearing collective responsibility," DEMATEL Report No. 1, Battelle Geneva Research Centre, Geneva, Switzerland, 1973.

[33] A. Özdemir and F. Tüysüz, “An integrated fuzzy DEMATEL and fuzzy ANP based balanced scorecard approach: application in Turkish higher education institutions," Journal of Multiple-Valued Logic and Soft Computing, vol. 28, no. 2-3, pp. 251-287, 2017.

[34] I.-S. Chen, "A revised Inno-Qual performance system for higher education: the integrated applications of DEMATEL and ANP," Journal of the Operational Research Society, vol. 63, no. 4, pp. 478-488, 2012.

[35] R. Ranjan, P. Chatterjee, and S. Chakraborty, "Evaluating performance of engineering departments in an Indian University using DEMATEL and compromise ranking methods," Opsearch, vol. 52, no. 2, pp. 307-328, 2015.

[36] G. Tzeng, C. Chiang, and C. Li, "Evaluating intertwined effects in e-learning programs: a novel hybrid MCDM model based on factor analysis and DEMATEL," Expert Systems with Applications, vol. 32, no. 4, pp. 1028-1044, 2007.
[37] J. S. Jeong and D. González-Gómez, “Adapting to PSTs' pedagogical changes in sustainable mathematics education through flipped E-learning: ranking its criteria with MCDA/ F-DEMATEL," Mathematics, vol. 8, no. 5, p. 858, 2020.

[38] C.-C. Chang and P.-Y. Chen, "Analysis of critical factors for social games based on extended technology acceptance model: a DEMATEL approach," Behaviour \& Information Technology, vol. 37, no. 8, pp. 774-785, 2018.

[39] J. K. Chen, "The influence of behavioural intention on thirdparty e-commerce payment," South African Journal of Economic and Management Sciences, vol. 21, no. 1, pp. 1-9, 2018.

[40] H. Y. Liao and J. K. Chen, "Comparison of acceptance in mobile smart wearable technology between SEM and DEMATEL methods," in Proceedings of the 2020 International Wireless Communications and Mobile Computing (IWCMC), pp. 1413-1417, IEEE, Limassol, Cyprus, June 2020.

[41] Y. C. Lee, Y. F. Hsieh, and Y. B. Guo, "Construct DTPB model by using DEMATEL: a study of a university library website," Program, vol. 47, no. 2, pp. 155-169, 2013.

[42] Y.-F. Hsieh, Y.-C. Lee, and S.-B. Lin, "Rebuilding DEMATEL threshold value: an example of a food and beverage information system," SpringerPlus, vol. 5, no. 1, p. 1385, 2016.

[43] S.-L. Si, X.-Y. You, H.-C. Liu, and P. Zhang, "DEMATEL technique: a systematic review of the state-of-the-art literature on methodologies and applications," Mathematical Problems in Engineering, vol. 2018, Article ID 3696457, 33 pages, 2018.

[44] L. A. Ocampo, T. A. G. Tan, and L. A. Sia, "Using fuzzy DEMATEL in modeling the causal relationships of the antecedents of organizational citizenship behavior (OCB) in the hospitality industry: a case study in the Philippines," Journal of Hospitality and Tourism Management, vol. 34, pp. 11-29, 2018.

[45] L. Ocampo and K. Yamagishi, "Modeling the lockdown relaxation protocols of the Philippine government in response to the COVID-19 pandemic: an intuitionistic fuzzy DEMATEL analysis," Socio-Economic Planning Sciences, vol. 72, Article ID 100911, 2020.

[46] J. Daniel, "Education and the COVID-19 pandemic," Prospects, vol. 49, no. 1, pp. 91-96, 2020.

[47] A. Shahzad, R. Hassan, A. Y. Aremu, A. Hussain, and R. N. Lodhi, "Effects of COVID-19 in E-learning on higher education institution students: the group comparison between male and female," Quality and Quantity, vol. 55, no. 3, pp. 805-826, 2021.

[48] L. Mishra, T. Gupta, and A. Shree, "Online teaching-learning in higher education during lockdown period of COVID-19 pandemic," International Journal of Educational Research Open, vol. 1, Article ID 100012, 2020.

[49] H.-H. Pham and T.-T.-H. Ho, "Toward a 'new normal' with e-learning in Vietnamese higher education during the post COVID-19 pandemic," Higher Education Research and Development, vol. 39, no. 7, pp. 1327-1331, 2020.

[50] S. Sukendro, A. Habibi, K. Khaeruddin et al., "Using an extended Technology Acceptance Model to understand students' use of e-learning during Covid-19: Indonesian sport science education context," Heliyon, vol. 6, no. 11, Article ID e05410, 2020.

[51] J. R. Bryson and L. Andres, "Covid-19 and rapid adoption and improvisation of online teaching: curating resources for extensive versus intensive online learning experiences," Journal of Geography in Higher Education, vol. 44, no. 4, pp. 608-623, 2020.

[52] Y. Chandra, "Online education during COVID-19: perception of academic stress and emotional intelligence coping 
strategies among college students," Asian Education and Development Studies, vol. 10, no. 2, pp. 229-238, 2021.

[53] A. Aristovnik, D. Keržič, D. Ravšelj, N. Tomaževič, and L. Umek, "Impacts of the COVID-19 pandemic on life of higher education students: a global perspective," Sustainability, vol. 12, no. 20, p. 8438, 2020.

[54] M. T. Hebebci, Y. Bertiz, and S. Alan, "Investigation of views of students and teachers on distance education practices during the Coronavirus (COVID-19) Pandemic," International Journal of Technology in Education and Science, vol. 4, no. 4, pp. 267-282, 2020.

[55] L. S. Neuwirth, S. Jović, and B. R. Mukherji, "Reimagining higher education during and post-COVID-19: challenges and opportunities," Journal of Adult and Continuing Education, 2020.

[56] O. B. Adedoyin and E. Soykan, "Covid-19 pandemic and online learning: the challenges and opportunities," Interactive Learning Environments, pp. 1-13, 2020.

[57] S. Brammer and T. Clark, "COVID-19 and management education: reflections on challenges, opportunities, and potential futures," British Journal of Management, vol. 31, no. 3, pp. $453-456,2020$.

[58] C. Azorín, "Beyond COVID-19 supernova. Is another education coming?" Journal of Professional Capital and Community, vol. 5, no. 3-4, pp. 381-390, 2020.

[59] J. Crawford, K. Butler-Henderson, J. Rudolph et al., "COVID19: 20 countries' higher education intra-period digital pedagogy responses," Journal of Applied Learning \& Teaching, vol. 3, no. 1, pp. 1-20, 2020.

[60] M. Assunção Flores and M. Gago, "Teacher education in times of COVID-19 pandemic in Portugal: national, institutional and pedagogical responses," Journal of Education for Teaching, vol. 46, no. 4, pp. 507-516, 2020.

[61] R. L. Quezada, C. Talbot, and K. B. Quezada-Parker, "From bricks and mortar to remote teaching: a teacher education program's response to COVID-19," Journal of Education for Teaching, vol. 46, no. 4, pp. 472-483, 2020.

[62] X. Zhu and J. Liu, "Education in and after Covid-19: immediate responses and long-term visions," Postdigital Science and Education, vol. 2, no. 3, pp. 695-699, 2020.

[63] T. Izumi, V. Sukhwani, A. Surjan, and R. Shaw, "Managing and responding to pandemics in higher educational institutions: initial learning from COVID-19," International Journal of Disaster Resilience in the Built Environment, vol. 12, no. 1, pp. 51-66, 2020.

[64] M. Händel, M. Stephan, M. Gläser-Zikuda, B. Kopp, S. Bedenlier, and A. Ziegler, "Digital readiness and its effects on higher education students' socio-emotional perceptions in the context of the COVID-19 pandemic," Journal of Research on Technology in Education, 2020.

[65] G. Grinberga Zalite and A. Zvirbule, "Digital readiness and competitiveness of the EU higher education institutions: the COVID-19 pandemic impact," Emerging Science Journal, vol. 4, no. 4, pp. 297-304, 2020.

[66] A. Joshi, M. Vinay, and P. Bhaskar, "Impact of coronavirus pandemic on the Indian education sector: perspectives of teachers on online teaching and assessments," Interactive Technology and Smart Education, 2020.

[67] F. J. García-Peñalvo, A. Corell, V. Abella-García, and M. Grande-de-Prado, "Recommendations for mandatory online assessment in higher education during the COVID-19 pandemic," in Radical Solutions for Education in a Crisis Context, Lecture Notes in Educational Technology, pp. 85-98, Springer, Singapore, 2020.
[68] N. Iivari, S. Sharma, and L. Ventä-Olkkonen, "Digital transformation of everyday life - how COVID-19 pandemic transformed the basic education of the young generation and why information management research should care?" International Journal of Information Management, vol. 55, Article ID 102183, 2020.

[69] C. Greenhow, C. Lewin, and K. B. Staudt Willet, "The educational response to Covid-19 across two countries: a critical examination of initial digital pedagogy adoption," Technology, Pedagogy and Education, vol. 30, no. 1, pp. 7-25, 2021.

[70] F. A. Azhari and L. C. Ming, "Review of e-learning practice at the tertiary education level in Malaysia," Indian Journal of Pharmaceutical Education and Research, vol. 49, no. 4, pp. 248-257, 2015.

[71] G. Vial, "Understanding digital transformation: a review and a research agenda," The Journal of Strategic Information Systems, vol. 28, no. 2, pp. 118-144, 2019.

[72] Y. Siron, A. Wibowo, and B. S. Narmaditya, "Factors affecting the adoption of e-learning in Indonesia: lesson from Covid19," Journal of Technology and Science Education, vol. 10, no. 2, pp. 282-295, 2020.

[73] W. H. DeLone and E. R. McLean, "The DeLone and McLean model of information systems success: a ten-year update," Journal of Management Information Systems, vol. 19, no. 4, pp. 9-30, 2003.

[74] C. Saxena, H. Baber, and P. Kumar, "Examining the moderating effect of perceived benefits of maintaining social distance on e-learning quality during COVID-19 pandemic," Journal of Educational Technology Systems, vol. 49, no. 4, pp. 532-554, 2021.

[75] H. Baber, "Modelling the acceptance of e-learning during the pandemic of COVID-19-A study of South Korea," International Journal of Management in Education, vol. 19, no. 2, Article ID 100503, 2021.

[76] M. M. Abbad, "Using the UTAUT model to understand students' usage of e-learning systems in developing countries," Education and Information Technologies, 2021.

[77] J. N. Jere, "Investigating university academics behavioural intention in the adoption of e-learning in a time of COVID19," South African Journal of Information Management, vol. 22, no. 1, pp. 1-9, 2020.

[78] S. Norris and F. Murphy, "A community of practice in a midwifery led unit. How the culture and environment shape the learning experience of student midwives," Midwifery, vol. 86, Article ID 102685, 2020.

[79] A. Tarhini, K. Hone, and X. Liu, "The effects of individual differences on e-learning users' behaviour in developing countries: a structural equation model," Computers in Human Behavior, vol. 41, pp. 153-163, 2014.

[80] R. A. Ancheta Jr, M. F. Bongo, L. A. Ocampo et al., "DEMATEL-AHP technique to minimise departure delays due to airspace congestion: a case in Mactan-Cebu International Airport," International Journal of System of Systems Engineering, vol. 8, no. 4, pp. 365-386, 2018. 